\title{
Protein Data Bank Japan: 20 years and more as the Asian hub for 3D structure and the founding member of the wwPDB
}

\author{
G. Kurisu \\ Institute for Protein Research, Osaka University, 3-2 Yamadaoka, Suita city, Osaka 565-0871, Japan \\ gkurisu@protein.osaka-u.ac.jp
}

Protein Data Bank Japan (PDBj) accepts and processes regional 3D structure data of biological macromolecules since 2000. We celebrated our 20th anniversary of our regional Data-in activities last year. Our Data-out service has a much longer history, dating back to before the establishment of PDBj. The first protein structure from Asia was determined at the Institute for Protein Research (IPR) in 1971 at $4 \AA$ [1] and a subsequent structure at $2.3 \AA$ solved in 1973 [2] was deposited to the PDB in 1975 as the 21 st entry in PDB. Based on these early contributions to the crystallographic community, IPR founded the Crystallographic Research Centre and installed several 4-circule diffractometers, and developed the Imaging Plate detectors of R-axis series later [3] together with Rigaku. In addition to above activities, IPR was assigned as the National Affiliated Centre of Cambridge Crystallographic Data Centre from 1978 and keep serving until now (http://www.protein.osaka-u.ac.jp/CSD/, Fig.1). Distribution of the PDB data from IPR started in 1979 as a regional data centre, initially by magnetic tape and later by CD-ROM, until the installation of an official mirror site of Brookhaven PDB in 1998. Since 2001, we have provided our newly developed online Data-out services freely and publicly through our own web site (https://pdbj.org; Fig.2), which includes our molecular graphics viewer, Molmil; a molecular surface database for functional sites, eFsite; and a database of protein dynamics calculated via normal mode analysis, Promode Elastic [4], and we have served since 2003 as a founding member of the worldwide PDB (https://wwpdb.org). During the COVID-19 pandemic, we have provided a COVID-19 featured page in three Asian languages (Japanese, Chinese and Korean) and have started a new service archiving raw X-ray image data directly related to deposited PDB entries (XRDa, https://xrda.pdbj.org; Fig.3) [5]. Since we already have BMRBj (formerly PDBj-BMRB) and EMPIAR-PDBj on-site, XRDa completes the regional experimental raw data archives of the related PDB, BMRB and EMDB entries from the three major experimental methods; Macromolecular Crystallography, NMR spectroscopy and 3D Electron Microscopy.
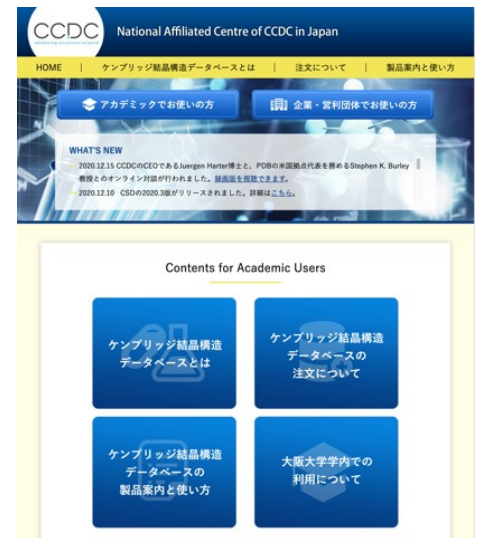

CCDC

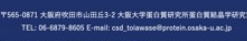

Fig 1. Web portal for the Japanese users of CSD

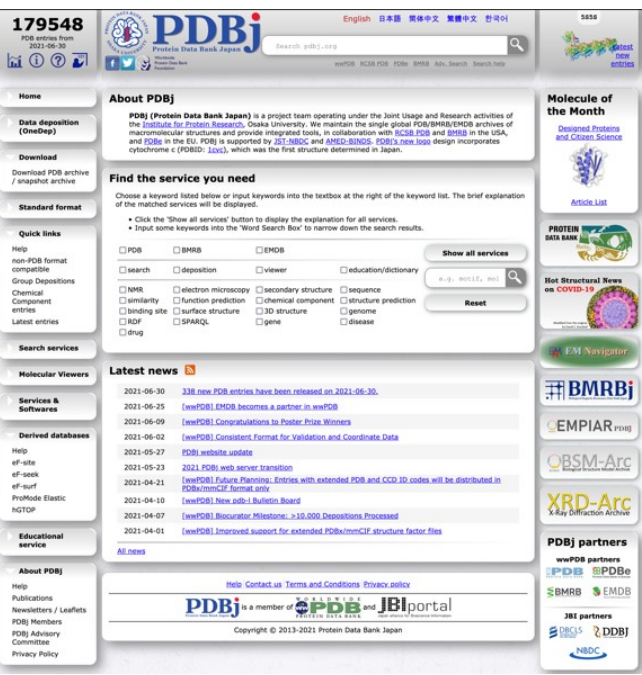

Fig 2. Main page of PDBj including the links to our services.

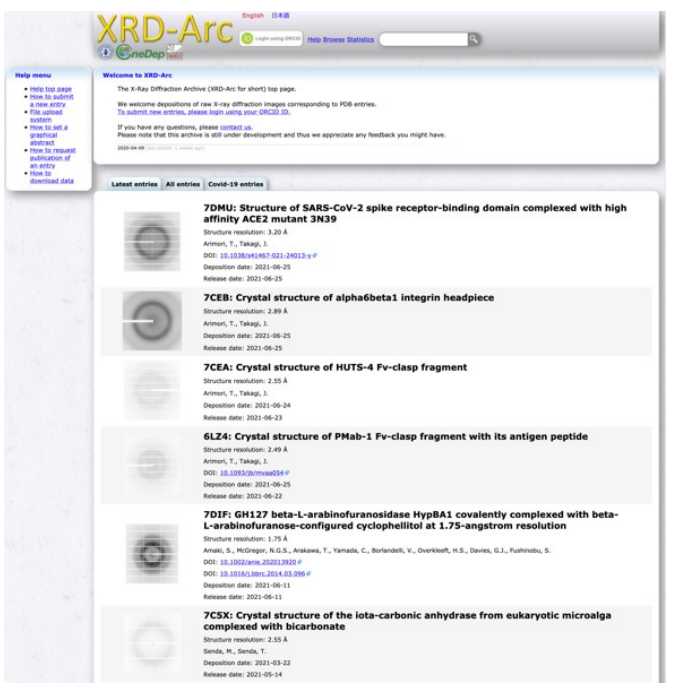

Fig 3. Web page of the XRDa.

[1] Ashida, T., Ueki, T., Tsukihara, T., Sugihara, A., Takano, T. \& Kakudo, M. (1971) J. Biochem. 70, $913-924$.

[2] Ashida, T., Tanaka, N., Yamane, T., Tsukihara, T. \& Kakudo, M. (1973) J. Biochem., 73, 463-465.

[3] Sato, M., Katsube, Y. \& Hayashi, K. (1993) J. Appl. Cryst., 26, 733-735.

[4] Kinjo, A.R., Bekker, G.-J., Wako, H., Endo, S., Tsuchiya, Y., Sato, H., Nishi, H., Kinoshita, K., Suzuki, H., Kawabata, T., Yokochi, M., Iwata, T., Kobayashi, N., Fujiwara, T., Kurisu, G. \& Nakamura, H. (2018) Protein Sci., 27, 95-102.

[5] Bekker, G.-J. \& Kurisu, G. in preparation

Keywords: Protein Data Bank; Cambridge Structural Database; worldwide PDB 